\title{
The Utility of Diagnostic Tests in Irritable Bowel Syndrome Patients: A Systematic Review
}

Brooks D. Cash, M.D., Philip Schoenfeld, M.D., M.S.Ed., M.Sc.(Epi.), and

William D. Chey, M.D., F.A.C.G., F.A.C.P.

Division of Gastroenterology, Uniformed Services University of the Health Sciences, Bethesda, Maryland; Division of Gastroenterology, University of Michigan School of Medicine, Ann Arbor, Michigan; and VA Center for Excellence in Health Care Research, Ann Arbor, Michigan

OBJECTIVE: The aim of this study was to determine the pretest probability of organic GI disease and the accuracy of diagnostic tests for organic GI disease in patients who meet symptom-based criteria for irritable bowel syndrome (IBS).

METHODS: After a comprehensive literature search for studies examining the accuracy of diagnostic tests for organic GI disease among patients who meet symptom-based criteria for IBS, two independent observers qualitatively assessed the methodology of selected studies and extracted data. Data on the pretest probability of organic GI disease in this population and the accuracy of currently recommended diagnostic tests were converted to descriptive tables.

RESULTS: Among patients meeting symptom-based criteria for IBS, the pretest probability of inflammatory bowel disease, colorectal cancer, or infectious diarrhea is less than $1 \%$. Currently recommended diagnostic tests rarely identify organic GI disease in patients fulfilling symptom-based criteria for IBS. However, the pretest probability of celiac disease in patients meeting symptom-based criteria for IBS was 10 times higher than the prevalence of celiac disease in the general population.

CONCLUSIONS: There is insufficient evidence to recommend the routine performance of a standardized battery of diagnostic tests in patients who meet symptom-based criteria for IBS. Based upon the increased pretest probability of celiac disease, routine performance of serological tests for celiac disease may be useful in this patient population, though additional study is needed in this area. (Am J Gastroenterol 2002;97:2812-2819. (C) 2002 by Am. Coll. of Gastroenterology)

\section{INTRODUCTION}

Irritable bowel syndrome (IBS) is a common chronic GI condition characterized by abdominal pain, bloating, and disturbed defecation. It is considered one of a group of functional GI disorders in which altered motility, abnormal visceral sensation, and psychosocial factors interplay to cause symptoms. The prevalence of IBS is estimated to be between $14 \%$ and $24 \%$ in women and $5-19 \%$ in men in the
United States and Britain (1). IBS is responsible for 2.4-3.5 million physician visits per year (2). Recent estimates of the total direct costs of IBS were \$1.6 billion in $1998(3,4)$. These data confirm that the management of IBS is a significant public health issue.

The symptoms of IBS are difficult to quantify. There is no reliable biological marker for the condition, requiring the use of symptom-based criteria for the diagnosis. Multiple symptom-based criteria (Table 1) have been developed, including the Manning, Rome I, and Rome II criteria (5-7). In particular, the Rome criteria, developed by multinational working groups, provide a uniform framework for the selection of patients in diagnostic and therapeutic trials of IBS. Of the commonly used symptom-based criteria for the diagnosis of IBS, the Manning criteria have been the most extensively studied. The positive predictive value of the Manning criteria for the diagnosis of IBS has ranged between $65-75 \%$, depending upon the number of symptoms present and the number of symptoms included for analysis (in the original Manning study, four of six criteria reached statistical significance as predictors of IBS, whereas two others approached statistical significance) (5).

In an attempt to build upon the strengths, while limiting the weaknesses of the Manning criteria, the Rome I criteria were developed largely through expert consensus. The Rome I criteria have been less extensively studied than the Manning criteria. In one trial that evaluated the diagnostic accuracy of the Rome I criteria in the absence of "red flag" symptoms, the sensitivity was $65 \%$, specificity $100 \%$, and positive predictive value $98 \%$ in distinguishing between IBS and organic disease (8). The Rome II criteria have not been formally validated but have been compared with the Manning and Rome I criteria in a number of studies. These studies have reported reasonably good agreement between the different criteria with $\kappa$ values of about 0.70 (9-11). It appears, however, that the Rome II criteria may be less sensitive than the Manning or Rome I criteria (12).

The Rome committee and many IBS authorities recommend that several diagnostic tests be performed as part of the evaluation of patients with suspected IBS $(13,14)$. The most recent American Gastroenterological Association Po- 
Table 1. Symptom-Based Criteria for the Diagnosis of IBS

\begin{tabular}{|c|c|c|}
\hline Manning Criteria & Rome Criteria & Rome II Criteria \\
\hline $\begin{array}{l}\text { Abdominal pain } \\
\text { relieved by } \\
\text { defecation }\end{array}$ & $\begin{array}{l}\text { At least } 12 \text { wk of continuous or recurrent symptoms } \\
\text { of the following: }\end{array}$ & $\begin{array}{l}\text { At least } 12 \mathrm{wk} \text {, which need not be consecutive, in } \\
\text { the preceding } 12 \text { mo of abdominal discomfort or } \\
\text { pain that has two of the three features: }\end{array}$ \\
\hline \multirow{3}{*}{$\begin{array}{l}\text { Looser stools with } \\
\text { the onset of pain }\end{array}$} & 1) relieved with defecation, or & \\
\hline & 2) associated with a change in frequency of stool, & \\
\hline & $\begin{array}{l}\text { 3) associated with a change in consistency of } \\
\text { stool }\end{array}$ & \\
\hline \multirow{3}{*}{$\begin{array}{l}\text { More frequent stools } \\
\text { with the onset of } \\
\text { pain }\end{array}$} & $\begin{array}{l}\text { Two or more of the following, at least on one } \\
\text { fourth of occasions or days: }\end{array}$ & $\begin{array}{l}\text { Onset associated with a change in frequency of } \\
\text { stool and/or }\end{array}$ \\
\hline & 1) altered stool frequency, or & \\
\hline & 2) altered stool form, or & \\
\hline \multicolumn{3}{|l|}{$\begin{array}{l}\text { Passage of mucous in } \\
\text { stools }\end{array}$} \\
\hline $\begin{array}{l}\text { Sensation of } \\
\text { incomplete } \\
\text { evacuation }\end{array}$ & & \\
\hline
\end{tabular}

sition Statement on IBS recommends the performance of a complete blood count (CBC), erythrocyte sedimentation rate (ESR), serum chemistries, and stool examination for ova and parasites (O\&P), and occult blood (FOBT). Colonic visualization with flexible sigmoidoscopy, barium enema, or colonoscopy (if the patient is older than $50 \mathrm{yr}$ of age) is also recommended (15). These evaluations are intended to rule out inflammatory bowel disease (IBD), colorectal cancer, and infectious diarrhea. Other experts also recommend the use of hydrogen breath tests and thyroid function testing $(16,17)$ to rule out lactose malabsorption and thyroid dysfunction, respectively. The degree to which these additional tests add to the diagnostic certainty of IBS, as established by validated symptom-based criteria such as the Manning or Rome criteria, is unknown. In other words, does the evidence support the routine application of additional diagnostic testing in patients who meet the Manning, Rome, or other symptom-based criteria for IBS?

Clinicians should consider several issues when deciding if a diagnostic test is needed, but two issues deserve particular attention. First, they should consider the pretest probability of the disorder based upon the known prevalence of the disorder in patients with specific symptoms. If the pretest probability of a disorder is miniscule, then further diagnostic tests may not be necessary (18). Second, clinicians should consider the accuracy (e.g., sensitivity, specificity, positive and negative predictive value) of the diagnostic test if the pretest probability of a disorder is high enough to warrant investigation. Therefore, a systematic review of the literature was undertaken to quantify the prevalence of organic GI disease and to determine the accuracy of the most commonly employed tests for the diagnosis of organic GI disease among patients who meet symptom-based criteria for IBS.

\section{MATERIALS AND METHODS}

\section{Literature Search}

A search of the online bibliographic databases MEDLINE and EMBASE was performed to identify all relevant articles published between 1980 and 2001. The search terms "colonic diseases, functional [diagnosis]" or "irritable, functional, or spastic" adjacent to "bowel or colon" were used. The search was further supplemented by the addition of other descriptive key words such as "blood," "parasite," "stool analysis," "radiography," "hydrogen breath testing," "flexible sigmoidoscopy or colonoscopy or barium enema." An expert medical librarian was used to maximize search techniques and retrieval of targeted information. The bibliographies from all potentially relevant articles were manually searched. Additionally, abstracts from the annual scientific meetings of the American Gastroenterological Association (Digestive Disease Week) and the American College of Gastroenterology (1998-2001) were reviewed.

\section{Study Selection Criteria}

Criteria for properly designed studies of diagnostic tests have been defined (19). These criteria include: 1) independent, blind comparison with a reference, or "gold" standard of diagnosis; 2) evaluation within an appropriate spectrum of patients where the diagnostic test is likely to be used; and 
Table 2. Qualitative Assessment of Selected Studies

\begin{tabular}{|c|c|c|c|c|c|c|c|c|}
\hline Reference & Population & Verification & $\begin{array}{l}\text { Interpretation } \\
\text { of Test }\end{array}$ & $\begin{array}{l}\text { Patient } \\
\text { Selection }\end{array}$ & $\begin{array}{c}\text { Data } \\
\text { Collection }\end{array}$ & $\begin{array}{l}\text { Details } \\
\text { of Test }\end{array}$ & $\begin{array}{c}\text { Details of } \\
\text { Reference Test }\end{array}$ & $\begin{array}{l}\text { Details of } \\
\text { Population }\end{array}$ \\
\hline 23 & +1 & 0 & 0 & +1 & +1 & +1 & 0 & +1 \\
\hline 24 & +1 & 0 & 0 & +1 & +1 & +1 & 0 & +1 \\
\hline 26 & +1 & 0 & 0 & +1 & +1 & +1 & 0 & +1 \\
\hline 27 & 0 & +1 & 0 & +1 & +1 & +1 & +1 & +1 \\
\hline 28 & +1 & 0 & 0 & +1 & +1 & 0 & +1 & +1 \\
\hline
\end{tabular}

Population: $+1=$ clinical; $0=$ case-control.

Verification: $+1=$ comparison of diagnostic test to "gold" standard or reference test; $0=$ no comparison of diagnostic test to reference test.

Interpretation of test: $+1=$ blinded interpretation; $0=$ nonblinded interpretation.

Patient selection: $+1=$ consecutive; $0=$ nonconsecutive.

Data collection: $+1=$ prospective; $0=$ nonprospective.

Details of test: $+1=$ sufficient details of diagnostic test applied; $0=$ insufficient details of diagnostic test applied.

Details of reference test: $+1=$ sufficient details of reference test used; $0=$ insufficient details of reference test used

Details of population: $+1=$ sufficient details regarding study and control populations; $0=$ insufficient details regarding study and control population.

3) application of the reference standard regardless of the diagnostic test result (i.e., elimination of verification bias).

To determine the accuracy of diagnostic evaluations in IBS patients, study selection criteria were: 1) use of a cohort of IBS patients explicitly diagnosed via symptom-based criteria (Manning, Rome I, Rome II, or International Congress of Gastroenterology criteria) (20); 2) performance of a commonly applied diagnostic test with a blinded comparison with an appropriate gold standard diagnostic test for organic GI disease; and/or 3) quantification of the results as either normal or abnormal in which an additional or alternative diagnosis of organic disease was made, based upon the test result. In all of the studies that fulfilled inclusion criteria, the diagnosis of IBS was made solely based upon fulfillment of symptom-based criteria and $a$ priori to the application of any of the diagnostic tests being evaluated.

Potentially relevant articles were reviewed in an independent, unblinded fashion by two authors (B.D.C., P.S.S.) to determine if they met the validity criteria specified above. Reviewers rated each article as being eligible, not eligible, or as having insufficient information to make a judgment as to eligibility. Any disagreement among reviewers was resolved by consensus.

\section{Qualitative Assessment of Study Methodology}

Previous reviews have established methodological criteria by which to determine the quality and validity of trials about diagnosis (21). The quality of each study selected for this systematic review was determined by assessing the following study characteristics: 1) study population; 2) verification; 3) interpretation of test results; 4) patient selection; 5) data collection; 6) details of test; 7) details of reference test; and 8) details of population (Table 2). The total score ranged from 0 to 8 . Studies were classified as low quality if they scored in the lowest tertile or as medium-high quality if they scored in the middle-to-high tertile, as described in previous reports (21).

\section{Data Extraction and Statistical Analysis}

Eligible articles were reviewed in a blinded fashion by two different investigators (B.D.C., P.S.S.), and the results of the primary research studies were abstracted onto specially designed data extraction forms. Data were extracted about: 1) population, including the proportion of female patients; 2) symptom-based criteria used (Manning, Rome I/II, International Congress of Gastroenterology); 3) intervention (diagnostic evaluation performed and gold standard comparison); and 4) outcome (prevalence of confirmed organic GI disease, resulting in an alternative diagnosis to explain IBS symptoms, and data on the diagnostic accuracy of tests). The frequency of agreement between reviewers was greater than $95 \%$, and disagreement was resolved through discussion.

To determine the pretest probability of organic GI disease in patients meeting symptom-based criteria for IBS, the prevalence of organic GI disease was extracted from study results and subsequently compared with the background prevalence based upon general population estimates. The sensitivity and specificity of each diagnostic test to accurately rule in or rule out a specific organic GI disease among this unique patient population was calculated when adequate data were provided to allow this calculation. Because of the wide variation of study methodology, study results were too diverse to combine in a true meta-analysis (22). Therefore, data are simply presented in tabular form.

\section{RESULTS}

\section{Characteristics of Selected Studies}

A total of 154 potentially relevant references were identified through the MEDLINE search, EMBASE search, citation search, and manual searches of abstracts from gastroenterology meetings. Reviews of the titles and abstracts followed by review of the full manuscripts of potentially relevant articles identified six published manuscripts (23-28) that met inclusion criteria. Selected characteristics and qualitative scores of the studies are included in Table 3. Two of the six studies $(25,27)$ scored in the highest tertile for methodological quality, and the remaining four studies scored in the median tertile for methodological quality. 
Table 3. Summary of Studies Selected

\begin{tabular}{|c|c|c|c|c|c|c|c|c|}
\hline Diagnostic Test & Reference & Gold Standard & $\begin{array}{c}\text { IBS } \\
\text { (n) }\end{array}$ & $\begin{array}{l}\text { Control } \\
\text { (n) }\end{array}$ & $\begin{array}{c}\text { Age } \\
\text { (Average) }\end{array}$ & $\begin{array}{l}\text { Gender } \\
\text { (F) } \\
(\%)\end{array}$ & $\begin{array}{c}\text { Prevalence of } \\
\text { Organic Disease } \\
(\%)\end{array}$ & Score \\
\hline \multirow[t]{4}{*}{ FS, BE, colonoscopy } & 23 & N/A & 306 & 0 & 45 & 72 & 1.31 & $5 / 8$ \\
\hline & 24 & N/A & 196 & 0 & 44 & 81 & 1.02 & $5 / 8$ \\
\hline & 25 & N/A & 89 & 59 & 44 & 82 & 0 & $6 / 8$ \\
\hline & 26 & N/A & 125 & 0 & 39 & 80 & 0 & $5 / 8$ \\
\hline Rectal biopsy & 25 & N/A & 89 & 59 & 44 & 82 & 0 & $6 / 8$ \\
\hline $\mathrm{CBC}$ & 27 & N/A & 300 & 300 & 56 & 71 & 0.33 & $6 / 8$ \\
\hline \multirow[t]{2}{*}{ Chemistries } & 24 & N/A & 196 & 0 & 44 & 81 & 1.02 & $5 / 8$ \\
\hline & 27 & N/A & 300 & 300 & 56 & 71 & 1.33 & $6 / 8$ \\
\hline AGA/EmA & 27 & Duodenal biopsy & 300 & 300 & 56 & 71 & 4.67 & $6 / 8$ \\
\hline FOBT & 24 & N/A & 183 & 0 & 44 & 81 & 0 & $5 / 8$ \\
\hline TSH & 23 & N/A & 1209 & 0 & 45 & 72 & 6 & $5 / 8$ \\
\hline \multirow{2}{*}{ Hydrogen breath test } & 24 & N/A & 186 & 0 & 44 & 81 & 25.8 & $5 / 8$ \\
\hline & 28 & N/A & 202 & 0 & 42 & 77 & 78 & $5 / 8$ \\
\hline Abdominal ultrasound & 26 & N/A & 125 & 0 & 39 & 80 & 0 & $5 / 8$ \\
\hline
\end{tabular}

$\mathrm{AGA}=$ anti-gliadin antibody $\mathrm{BE}=$ barium enema $\mathrm{EmA}=$ flexible endomysial antibody $; \mathrm{FS}=$ flexible sigmoidoscopy; $/ \mathrm{A}=$ not applicable.

\section{Pretest Probability of Organic GI Disease}

The pretest probability of various organic GI disease states in patients meeting symptom-based criteria for IBS is presented in Table 4. These data were extracted from the selected published manuscripts that fulfilled study selection criteria (23-28). For comparison purposes, prevalence rates of these organic GI diseases in the general population are also included. The pretest probability of IBD, colon cancer, lactose malabsorption, and thyroid dysfunction in patients with suspected IBS was similar to the prevalence of these disorders in the general population. However, the pretest probability of celiac disease was 10 times greater in patients with suspected IBS compared with the estimated prevalence of celiac disease in the general population.

\section{Colonic Evaluation for IBD, Other Forms of Colitis, and Colorectal Cancer}

Several studies (23-26) have systematically evaluated the structure of the colon with a variety of testing methods to

Table 4. Pretest Probability of Organic GI Disease in Patients Meeting Symptom-Based Criteria for IBS

\begin{tabular}{|c|c|c|}
\hline Organic GI Disease & $\begin{array}{c}\text { IBS Patients } \\
\text { (Pretest Probability) } \\
(\%)\end{array}$ & $\begin{array}{c}\text { General Population } \\
\text { (Prevalence) } \\
(\%) \\
\end{array}$ \\
\hline D & $51-$ & \\
\hline cancer & & \\
\hline ella & 4.6 & $0.25-0.5 \dagger$ \\
\hline al infection & $0-1.7$ & N/A $\ddagger$ \\
\hline 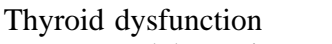 & 6 & $5-9 \S$ \\
\hline Lactose malabsorption & $22-26$ & 25 II \\
\hline \multicolumn{3}{|c|}{$\begin{array}{l}* \text { Reference } 4 . \\
\quad \nmid \text { Reference } 34 \text {. } \\
\quad \text { Reference } 4 \text { (unable to quantify point prevalence, but estimates approach annual } \\
\text { incidence of } 10-15 \% \text { of U.S. population). } \\
\text { \& Reference } 29 \text {. } \\
\text { I Reference } 30 .\end{array}$} \\
\hline
\end{tabular}

identify IBD, colitis, or colorectal cancer among patients with symptoms of IBS. Hamm et al. (23) examined the use of flexible sigmoidoscopy, colonoscopy, or barium enema in patients with suspected IBS fulfilling the Rome I criteria. It is not clear how many subjects underwent each individual examination. Among 306 patients studied, four were given alternative diagnoses (three IBD, one colonic obstruction), which may have been responsible for their IBS symptoms. Tolliver et al. performed a similar analysis in 196 subjects with suspected IBS (24). Like the study by Hamm et al. (23), the percentage of the cohort that underwent each examination is unclear. Forty-two colonic structural abnormalities were found in 34 subjects. Of these 42 abnormalities, two patients were found to have organic GI diseases (one IBD, one cancer) that could have been potential causes of IBS symptoms. The remainder of the abnormalities consisted of benign polyps, diverticulosis, hemorrhoids, lipomata, and melanosis coli.

MacIntosh et al. evaluated flexible sigmoidoscopy in patients with suspected IBS and in non-IBS controls (25). Among the IBS cohort, 89\% fulfilled the Manning criteria, and $84 \%$ fulfilled the Rome I criteria, whereas $15 \%$ of the controls fulfilled the Manning criteria, and only 5\% fulfilled the Rome I criteria. No patients with suspected IBS were given alternative diagnoses to explain their GI symptoms based upon flexible sigmoidoscopy in this trial. Francis et al. performed colonic examination with flexible sigmoidoscopy, barium enema, or colonoscopy in 125 patients who fulfilled the Rome I criteria for IBS (26). Except for incidental diverticular disease, no organic GI disorders were identified through the performance of these tests, and no patients were given alternative diagnoses to explain their GI symptoms as a result of these examinations.

Only one study meeting inclusion criteria evaluated the 
use of rectal biopsies as part of the evaluation of suspected IBS (25). No patients from among a group of 89 patients with suspected IBS or 59 non-IBS controls had rectal biopsy findings that resulted in an alternative or additional diagnosis of organic GI disease.

\section{Laboratory Evaluations (CBC, Chemistries, ESR/CRP, FOBT, Antigliadin/Endomysial Antibodies) for Identification of IBD, Other Colitides, Colorectal Cancer, or Celiac Disease}

Several reports $(24,27)$ have examined the use of commonly recommended laboratory tests such as CBC, serum chemistries, and FOBT as part of the diagnostic evaluation of suspected IBS. In the trial by Tolliver et al., CBC and serum chemistries were performed in 196 patients with suspected IBS (24). In no patient did the CBC result in an alternative diagnosis of organic GI disease. Serum chemistries were abnormal in two patients $(1.0 \%)$. Both subjects had abnormal liver-associated enzymes. The etiology of these abnormalities is not revealed in the article, so it is unclear whether or not they represented an alternative diagnosis of organic GI disease (responsible for the patients' IBS symptoms), or if they were indicative of a second disorder in addition to IBS.

Sanders $e$ t al. evaluated the use of CBC and serum chemistries in 300 patients who fulfilled the Rome II criteria for IBS (27). They identified five patients (1.67\%) who had organic GI disease. One patient was anemic and later found to have celiac disease, and two patients had abnormal liverassociated enzymes that were attributed to excess alcohol intake, as were their GI symptoms. The effect of cessation of alcohol intake upon GI symptoms in these patients was not reported. Two patients with elevated c-reactive protein (CRP), one of who also had an elevated ESR, were found to have IBD. These investigators also performed antigliadin antibody (IgA and $\operatorname{IgG}$ ) and endomysial antibody testing in 300 patients with suspected IBS and in 300 age- and gendermatched asymptomatic controls. Positive antibody tests were followed by upper endoscopy and distal duodenal biopsies. Sixty-six patients (22\%) with suspected IBS had positive antibody tests, and $14(4.67 \%)$ had histological evidence of celiac disease compared with two $(0.67 \%)$ controls. The effect of a gluten-free diet upon the GI symptoms of patients diagnosed with celiac disease was not reported in this study.

The study by Tolliver et al. was the only one to examine the use of FOBT in patients with suspected IBS (24). Fifteen of 183 patients $(8.2 \%)$ had a positive FOBT and subsequently underwent full colonoscopic examination. Four of the 15 with positive FOBT, or $2.2 \%$ of the original cohort, had structural abnormalities identified during colonoscopy. None of these findings were believed to represent an alternative diagnosis to IBS or provide an explanation for the patients' IBS symptoms.

\section{Stool Analysis for $O \& P$}

Examination of stool for O\&P is commonly recommended as a primary examination in the evaluation of patients with suspected IBS. Two trials have evaluated the results of stool O\&P examination in this population. Hamm et al. (23) found that $1.7 \%$ (19 of 1154) of patients with suspected IBS had evidence of an intestinal pathogen on standard stool O\&P examination. Of these 19 subjects, eight $(0.69 \%)$ were colonized with Blastocystis hominis, a relatively common finding of unclear clinical significance (23). Clinical outcomes after eradication of the identified pathogens were not reported (appropriate gold standard for this diagnosis would be resolution of IBS symptoms after successful eradication of infectious agent). Likewise, Tolliver et al. performed stool O\&P examinations in 170 patients with suspected IBS and found no subjects with evidence of enteric infection (24).

\section{Serological Testing for the Identification of Thyroid Dysfunction (TSH)}

Two studies have evaluated the role of TSH as part of the diagnostic evaluation of IBS. Hamm et al. performed TSH in more than 1200 patients fulfilling the Rome I criteria and identified 67 patients (6\%) with thyroid function abnormalities (23). These abnormalities were evenly distributed between hyper- and hypothyroidism. It is not clear whether these thyroid abnormalities identified were responsible for the patients' IBS symptoms because there is no report of symptom relief in response to correction of thyroid function (again, the ultimate gold standard for hyper- or hypothyroidism as the etiology of IBS would be resolution of IBS-type symptoms after treatment of the thyroid disorder). Tolliver et al. identified one of 171 patients with suspected IBS $(0.6 \%)$ who had an abnormal TSH (24). The nature of the thyroid dysfunction was not included in the text of the article, nor was the impact of therapy for the thyroid abnormality upon GI symptoms. These data must be considered carefully, however, because thyroid function test abnormalities are common in the general population with an expected prevalence of 5-9\% (29).

\section{Hydrogen Breath Testing for the Identification of Lactose Intolerance or Small Intestinal Bacterial Overgrowth (SIBO)}

The prevalence of lactose malabsorption (diagnosed via abnormal hydrogen breath testing) is estimated to be approximately $25 \%$ in western countries and as high as $75 \%$ worldwide $(30,31)$. Two trials have reported the results of hydrogen breath testing for lactose malabsorption in patients with suspected IBS $(23,24)$. One trial found that $23 \%$ (256 of 1122) of patients with suspected IBS, when administered a 25-g lactose dose, demonstrated impaired lactose absorption (23). Response to a lactose-free diet was not reported, so it is impossible to determine how many of these subjects had GI symptoms caused by lactose malabsorption rather than IBS. In another study, 186 patients with suspected IBS 
were evaluated with hydrogen breath testing (50-g dose of lactose) (24). These investigators found a similar prevalence of lactose malabsorption with $25.8 \%$ (48 of 186) of the cohort having abnormal results. In a subsequent publication, reflecting $3 \mathrm{yr}$ of follow-up, these investigators demonstrated that patients with suspected IBS diagnosed with lactose malabsorption did not differ with regards to their GI symptoms when compared with patients without hydrogen breath test evidence of lactose malabsorption (32).

Pimentel et al. performed lactulose hydrogen breath testing in 202 patients fulfilling the Rome I criteria referred for evaluation of possible SIBO (28). Seventy-eight percent (157 of 202) had breath test results that were consistent with SIBO and were treated with a 10-day course of antibiotics. Forty-seven of these patients (29.9\%) were restudied 7-10 days after completion of the antibiotics, and SIBO eradication was achieved in $25(53.2 \%)$. Twelve patients (48\%) with SIBO eradication did not meet the Rome I criteria when their symptoms were reassessed, whereas only four patients $(18.2 \%)$ with persistent SIBO failed to meet the Rome I criteria after treatment.

\section{Imaging Studies}

Francis et al. evaluated the role of abdominal ultrasound to identify serious abdominal or pelvic pathology in 125 patients (100 women, 25 men) with suspected IBS by the Rome I criteria (26). Twenty percent of women and $8 \%$ of men had an abnormality of some form found on abdominal ultrasound examination. Ten percent of women had pelvic abnormalities, the majority of which were gynecological in origin. The prevalence of hepatobiliary abnormalities was similar in both women and men (10\% and $8 \%$, respectively). Importantly, the identification of an anatomic abnormality on ultrasound did not lead to additional therapeutic measures in any patient, nor were the authors able to correlate any of the abnormalities identified on ultrasound to the patients' GI symptoms. These investigators concluded that abdominal ultrasound in patients with the "positive diagnosis of IBS" via symptom-based criteria was not necessary and may actually be counterproductive because the identification of trivial anatomic abnormalities could conceivably lead to unnecessary patient concern and additional, more invasive tests or procedures.

\section{DISCUSSION}

This systematic review of the trials examining the most commonly used diagnostic tests ordered in patients with suspected IBS identified two important points for discussion. First, there are very few rigorously designed trials examining the pretest probability and accuracy of diagnostic tests in patients fulfilling symptom-based criteria for IBS. Second, the available data demonstrate that patients fulfilling symptom-based criteria for IBS have very low pretest probabilities of organic GI disease, with the possible exception of celiac disease, and that most diagnostic testing in this population may be unnecessary. In fact, the pretest probability of organic disease in patients with suspected IBS who fulfill symptom-based criteria and do not demonstrate "alarm" symptoms is so low that a standardized battery of diagnostic tests to rule out organic disease may not be necessary.

Based upon the available data, the pretest probability of identifying organic GI disease responsible for IBS symptoms through colonic visualization with flexible sigmoidoscopy, barium enema, or colonoscopy is less than $1 \%$. Most identified colonic abnormalities are asymptomatic (e.g., polyps, diverticulosis) (33). The performance of rectal biopsies also failed to generate alternative diagnoses in the one prospective study that examined its use (25). Abdominal imaging via ultrasound examination also failed to detect any serious GI pathology or result in a change of the diagnosis or management of IBS in the one study that evaluated its use (26).

Data regarding the use of standard laboratory tests such as $\mathrm{CBC}$, serum chemistries, TSH, ESR/CRP, stool O\&P, and FOBT are similarly lacking. In the two trials that included CBC as part of the evaluation of patients with suspected IBS, only one patient was found to have an organic GI disease as a consequence of performing this test $(24,27)$. Both studies reported that performance of routine serum chemistries was similarly disappointing. Performance of TSH and stool examinations for O\&P and occult blood also failed to identify a significantly greater degree of organic GI disease in patients with suspected IBS compared with historical prevalence rates in the general population.

One study reported a significantly higher pretest probability for celiac disease in patients with suspected IBS compared with controls and historical prevalence rates (27, 34). Unfortunately, response to a gluten-free diet was not reported in this study. In an ideal study, patients diagnosed with celiac disease would have been treated with a glutenfree diet and followed to determine if this intervention was associated with resolution of their IBS symptoms. Recently, Wahnschaffe et al. examined the prevalence of celiac disease in a group of patients with suspected IBS and found that human leukocyte antigen subtype DQ2 genotype, duodenal biopsies with increased intraepithelial lymphocytes, and duodenal aspirates with elevated IgA antigliadin antibodies all supported the diagnosis of celiac disease in 23$35 \%$ of these patients (35). This study was not included in our formal systematic review, as it did not define IBS patients using formal symptom-based criteria. Surprisingly, none of the patients with suspected IBS diagnosed with celiac disease in this study had elevated serum antigliadin antibodies or tissue transglutaminase antibodies. A glutenfree diet was associated with improved GI symptoms in a subset of patients with suspected IBS diagnosed with celiac disease, but only 26 patients were studied. Therefore, this study suggests that serum antibody testing for celiac disease has a highly variable sensitivity, which appears to be at least in part related to the severity of intestinal injury $(36,37)$. 
Nevertheless, the identification of celiac disease is quite attractive given the availability of effective therapy and the ability of this therapy to prevent significant long-term morbidity. Thus, diagnostic testing for celiac disease may be considered routinely in patients who fulfill symptom-based criteria for IBS.

There is also some evidence that SIBO may play a role in a subset of those patients with symptoms attributed to IBS. However, well-designed clinical trials with appropriate treatment and adequate follow-up are necessary before adopting this association as part of the clinical mainstream. The results of the one report included in this review must be interpreted with caution because of the use of a suboptimal gold standard for SIBO, open label diagnosis and treatment, and the low rate of follow-up testing and documentation of treatment effect in only a small number of patients (28). Such evaluation was carried out in only $30 \%$ (47 of 157) of the original study cohort. Follow-up was also limited with a one-time reassessment of GI symptoms within 7-10 days after antibiotic treatment. Long-term, repeated reassessment of symptom-based IBS criteria and SIBO status in a larger number of patients would help strengthen the possible link between SIBO and IBS.

The community adherence to the currently recommended diagnostic evaluation of IBS is not known. However, it is known that additional diagnostic testing to exclude organic GI disease does contribute to the significant economic burden associated with this condition $(3,4)$. At first glance, based upon the results of this systematic review, the low pretest probability of organic GI disease among patients who meet symptom-based criteria for IBS argues against an exhaustive diagnostic evaluation for organic GI disease. However, several points regarding this conclusion merit discussion. A diagnostic evaluation is indicated if the patient has "alarm" symptoms, such as age $\geq 50 \mathrm{yr}$, weight loss, gross hematochezia, or systemic signs of infection or colitis. Additionally, the true value, for both patients with suspected IBS and their physicians, of a negative diagnostic evaluation might derive from the reassurance that they do not have an organic GI disease. Such reassurance from a negative evaluation may have beneficial effects upon symptom response regardless of any therapeutic intervention undertaken by the health care provider. Unfortunately, such an intangible and subjective outcome is difficult to measure and may be equally achievable through a well-developed patient-physician relationship. Much of the data regarding the diagnostic evaluation of patients with suspected IBS originate from secondary and tertiary referral centers, and the apparent pretest probability of organic GI disease in patients from these referral sites may be misrepresented as a result of this potential bias. Lastly, the average age of the patients in all of the studies reviewed ranged from 39 to $56 \mathrm{yr}$, so the pretest probability of organic diseases that are more prevalent with advanced age, such as colon cancer, would be expected to be lower in these cohorts than historical controls.
Future research in this area is needed and should address the following points. First, what is the overall rate of compliance with published clinical practice guidelines for the diagnosis of IBS? Second, given the lack of available data, there is a need for additional large, well-designed prospective trials (38) with adequate follow-up to examine the yield and accuracy of commonly used diagnostic tests obtained as part of the evaluation of IBS. If organic GI disease is diagnosed, then patients should be followed, after appropriate treatment, with periodic reassessment of their IBS symptoms to determine if treatment of the organic GI disease significantly impacts upon their clinical course. The findings of this systematic review have additional implications for the design of future IBS trials. Our results suggest that performing rigorous, invasive, and expensive diagnostic evaluations in potential study participants who clearly fulfill symptom-based criteria for IBS may not be necessary.

\section{ACKNOWLEDGMENTS}

This research was supported by an unrestricted educational grant from Novartis Pharmaceuticals Corporation.

The opinions and assertions contained herein are the sole views of the authors and should not be construed as official or as representing the views of the U.S. Navy, Department of Defense, or Department of Veteran Affairs.

Reprint requests and correspondence: Brooks D. Cash, M.D., Chief, Division of Gastroenterology, Naval Hospital Camp Lejeune, 100 Brewster Avenue, Camp Lejeune, NC 28547.

Received Apr. 15, 2002; accepted June 24, 2002.

\section{REFERENCES}

1. Drossman DA, Whitehead WE, Camilleri M. Irritable bowel syndrome: A technical review for practice guideline development. Gastroenterology 1997;112:2120-37.

2. Sandler RS. Epidemiology of irritable bowel syndrome in the United States. Gastroenterology 1990;99:409-15.

3. Talley NJ, Gabriel SE, Harmsen WS, et al. Medical costs in community subjects with irritable bowel syndrome. Gastroenterology 1995;109:1736-41.

4. Goodman C, Cronin K, Gemmen E, et al. In: The burden of gastrointestinal diseases. Bethesda, MD: American Gastroenterological Association, 2001:2-65.

5. Manning AP, Thompson WG, Heaton KW, et al. Towards a positive diagnosis of the irritable bowel syndrome. Br Med J 1978;2:653-4.

6. Thompson WG, Dotewall G, Drossman DA, et al. Irritable bowel syndrome: Guidelines for the diagnosis. Gastroenterol Int 1989;2:92-5.

7. Thompson WG, Longstreth GF, Drossman DA, et al. Functional bowel disorders and functional abdominal pain. Gut 1999;45(suppl II):II43-II47.

8. Vanner SJ, Depew WT, Paterson WG, et al. Predictive value of the Rome criteria for diagnosing the irritable bowel syndrome. Am J Gastrenterol 1999;94:2803-7.

9. Saito YA, Locke GR, Talley NJ, et al. A comparison of the Rome and Manning criteria for case identification in epidemi- 
ological investigations of irritable bowel syndrome. Am J Gastroenterol 2000;95:2679-81.

10. Thompson WG, Irvine EJ, Pare P, et al. Functional gastrointestinal disorders in Canada: First population-based survey using Rome II criteria with suggestions for improving the questionnaire. Dig Dis Sci 2002;47:225-35.

11. Mearin F, Badia X, Balboa A, et al. Irritable bowel syndrome prevalence varies enormously depending upon the employed diagnostic criteria: Comparison of Rome II versus previous criteria in a general population. Scand J Gastroenterol 2001; 36:1121-2.

12. Chey WD, Olden K, Carter E. Utility of the Rome I and Rome II criteria for IBS in US women. Am J Gastroenterol 2002 (in press).

13. Drossman DA. An integrated approach to the irritable bowel syndrome. Aliment Pharmacol Ther 1999;13(suppl 2):3-14.

14. Camilleri M, Prather CM. The irritable bowel syndrome: Mechanisms and a practical approach to management. Ann Intern Med 1992;116:1001-8.

15. American Gastroenterological Association Medical Position Statement. Irritable bowel syndrome. Gastroenterology 1997; 112:2118-9.

16. Schmulson MW, Chang L. Diagnostic approach to the patient with irritable bowel syndrome. Am J Med 1999;107:20S-26S.

17. Camilleri M. Management of the irritable bowel syndrome. Gastroenterology 2001;120:652-68.

18. Schoenfeld PS, Guyatt G, Hamilton F, et al. An evidencebased approach to gastroenterology diagnosis. Gastroenterology 1999;116:1230-7.

19. Jaeschke R, Guyatt GH, Sackett DL. Users guide to the medical literature. III. How to use an article about a diagnostic test. A. Are the results of the study valid? Evidence based medicine working group. JAMA 1994;271:389-91.

20. Hasler WL, Owyang C. Irritable bowel syndrome. In: Yamada T, ed. Textbook of gastroenterology. Philadelphia: JB Lippincott, 1991:1696-716.

21. Lijmer JG, Mol BW, Heisterkamp S, et al. Empirical evidence of design-related bias in studies of diagnostic tests. JAMA 1999;282:1061-6.

22. Irwig L, Tostetson AN, Gastonis C, et al. Guidelines for meta-analyses evaluating diagnostic tests. Ann Intern Med 1994;120:667-76.

23. Hamm LR, Sorrells SC, Harding JP, et al. Additional investigations fail to alter the diagnosis of irritable bowel syndrome in subjects fulfilling the Rome criteria. Am J Gastroenterol 1999;94:1279-82.

24. Tolliver BA, Herrera JL, DiPalma JA. Evaluation of patients who meet clinical criteria for irritable bowel syndrome. Am J Gastroenterol 1994;89:176-8.

25. MacIntosh DG, Thompson WG, Patel DG, et al. Is rectal biopsy necessary in irritable bowel syndrome? Am J Gastroenterol 1992;87:1407-9.

26. Francis CY, Duffy JN, Whorwell PJ, et al. Does routine ultrasound enhance diagnostic accuracy in irritable bowel syndrome? Am J Gastroenterol 1996;91:1348-50.

27. Sanders DS, Carter MJ, Hurlstone DP, et al. Association of adult coeliac disease with irritable bowel syndrome: A casecontrol study in patients fulfilling the ROME II criteria referred to secondary care. Lancet 2001;358:1504-8.

28. Pimentel M, Chow EJ, Lin HC. Eradication of small intestinal bacterial overgrowth reduces symptoms of irritable bowel syndrome. Am J Gastroenterol 2000;95:3503-6.

29. Helfand M, Redfern CC. Screening for thyroid disease: An update. Ann Intern Med 1998;129:144-58.

30. Scrimshaw NS, Murray EB. The acceptability of milk and milk products in populations with a high prevalence of lactose intolerance. Am J Clin Nutr 1988;48(suppl 4):1079-159.

31. Bourlioux P, Pochart P. Nutritional and health properties of yogurt. World Rev Nutr Diet 1988;56:217-58.

32. Tolliver BA, Jackson MS, Jackson KL, et al. Does lactose maldigestion really play a role in the irritable bowel syndrome? J Clin Gastroenterol 1996;23:15-7.

33. Otte JJ, Larsen L, Andersen JR. Irritable bowel syndrome and symptomatic diverticular disease-Different diseases. Am J Gastroenterol 1986;81:529-31.

34. Ciclitira PJ. AGA technical review on celiac sprue. Gastroenterology 2001;120:1526-40.

35. Wahnschaffe U, Ullrich R, Riecken EO, et al. Celiac diseaselike abnormalities in a subgroup of patients with irritable bowel syndrome. Gastroenterology 2001;121:1329-38.

36. Rostami K, Kerckhaert J, Tiemessen R, et al. Sensitivity of antiendomysium and antigliadin antibodies in untreated celiac disease: Disappointing in clinical practice. Am J Gastroenterol 1999;94:888-94.

37. Tursi A, Brandimarte G, Giorgetti G, et al. Low prevalence of antigliadin and anti-endomysium antibodies in subclinical/ silent celiac disease. Am J Gastroenterol 2001;96:1507-10.

38. Hawkey CJ. Irritable bowel syndrome clinical trial design: Future needs. Am J Med 1999;107:98S-102S. 